\title{
Maximal rank of extremal marginal tracial states
}

\author{
Hiromichi Ohno ${ }^{\text {a) }}$ \\ Department of Mathematics, Faculty of Engineering, Shinshu University, 4-17-1 Wakasato, \\ Nagano 380-8553, Japan
}

(Received 10 December 2009; accepted 31 July 2010; published online 23 September 2010)

States on the coupled quantum system $M_{n}(\mathrm{C}) \otimes M_{n}(\mathrm{C})$ whose restrictions to each subsystem are the normalized traces are called marginal tracial states. We investigate extremal marginal tracial states and compute their maximal rank. Diagonal marginal tracial states are also considered. (C) 2010 American Institute of Physics. [doi:10.1063/1.3481567]

\section{INTRODUCTION}

States on coupled quantum systems have recently studied from many points of view. In this paper, we focus on states on the coupled quantum system $M_{n}(\mathrm{C}) \otimes M_{n}(\mathrm{C})$ whose restrictions to each subsystem are the normalized traces. Such states are called marginal tracial states. In Ref. 2, Arveson showed a marginal tracial state of rank $r$ is almost surely entangled when $r \leqslant n / 2$.

In Ref. 5, Parthasarathy showed that every extremal marginal tracial state on $M_{2}(\mathrm{C})$ $\otimes M_{2}(\mathrm{C})$ is a pure state. After that, in Ref. 6, Price and Sakai gave necessary and sufficient conditions for a state to be extremal among marginal tracial states and conjectured that every such a state is pure if $n \geq 3$. Actually, in classical probability theory, the Birkhoff's theorem says that every extremal point of the set of doubly stochastic matrices of order $n$ is a permutation matrix, so that the only extremal points are the trivial ones. In this paper, by using the one-to-one correspondence between marginal tracial states on $M_{n}(\mathrm{C}) \otimes M_{n}(\mathrm{C})$ and unital completely positive trace preserving (UCPT) maps on $M_{n}(\mathrm{C})$ (see, e.g., Refs. 1, 7, and 8), we give a negative answer to the conjecture with the help of the result of Landau and Streater (Ref. 4, Theorem 1) which shows the existence of a nonunitary extremal UCPT map on $M_{n}(\mathrm{C})$ for $n \geq 3$. To the author's knowledge, this result, first known by Arveson and told to the author by Price in private, has never been published, and so we will give it for the completeness of the paper. Moreover, we compute the maximal rank of extremal marginal tracial states for some special cases and consider diagonal marginal tracial states which correspond to diagonal UCPT maps introduced in Ref. 4.

In Sec. II, we recall the relation between marginal tracial states and UCPT maps and construct a nonpure extremal marginal tracial state. Furthermore, we compute the maximal rank of extremal marginal tracial states on $M_{n}(\mathrm{C}) \otimes M_{n}(\mathrm{C})$ for $n=3,4$ and give a lower bound on the maximal rank of extremal marginal tracial states for $n \geq 5$. In Sec. III, we investigate the maximal rank of extremal diagonal marginal tracial states and show that the set of such states of rank $a$ with $a^{2}$ $\leq n$ is dense in the set of all diagonal marginal tracial states of rank $a$ or less.

\section{MARGINAL TRACIAL STATES AND UCPT MAPS}

In this section we recall the relation between marginal tracial states and UCPT (unital completely positive trace preserving) maps and compute the maximal rank of extremal marginal tracial states.

Definition 2.1: A state $\rho$ on $M_{n}(\mathrm{C}) \otimes M_{n}(\mathrm{C})$ is a marginal tracial state if the restrictions of $\rho$ to $M_{n}(\mathrm{C}) \otimes I$ and $I \otimes M_{n}(\mathrm{C})$ are the normalized traces. Denote by $\Gamma(n)$ the set of all marginal tracial states on $M_{n}(\mathrm{C}) \otimes M_{n}(\mathrm{C})$.

\footnotetext{
${ }^{\text {a)} E l e c t r o n i c ~ m a i l: ~ h \_o h n o @ s h i n s h u-u . a c . j p . ~}$
} 
Extremal marginal tracial states are considered in Refs. 5 and 6, and they are all pure states, if $n=2 .{ }^{5}$ We will show that this is not the case when $n \geq 3$ by using the one-to-one correspondence between marginal tracial states and UCPT maps.

Denote by $\operatorname{UCPT}(n)$ the set of all UCPT maps on $M_{n}(\mathrm{C})$. Every map $\varphi \in U C P T(n)$ can be written as

$$
\varphi(A)=\sum_{i=1}^{k} v_{i}^{*} A v_{i}
$$

for some $\left\{v_{i}\right\} \subset M_{n}(\mathrm{C})$ with $\sum_{i=1}^{k} v_{i}^{*} v_{i}=\sum_{i=1}^{k} v_{i} v_{i}^{*}=I$. The choice of the matrices $\left\{v_{i}\right\}$ is not unique, but they can be taken linearly independent. Thus, the number $k$ of the terms is uniquely determined (see Ref. 3) and is denoted by $r(\varphi)=k$.

For any $\varphi \in U C P T(n)$, the map $\varphi \otimes \mathrm{id}_{M_{n}(\mathrm{C})}$ is a unital positive map, so that the composition of $\varphi \otimes \mathrm{id}_{M_{n}(\mathrm{C})}$ with any state on $M_{n}(\mathrm{C}) \otimes M_{n}(\stackrel{n}{\mathrm{C}})$ is also a state. Let, in particular, choose the pure marginal tracial state given by the vector $\xi=\frac{1}{\sqrt{n}} \sum_{i=1}^{n} e_{i} \otimes e_{i} \in \mathrm{C}^{n} \otimes \mathrm{C}^{n}$ with a fixed orthonormal basis $\left\{e_{i}\right\}$ of $\mathrm{C}^{n}$. Then the composition is a marginal tracial state. Indeed, it easily follows from the equation

$$
\operatorname{tr}\left(e_{i j}{ }^{t} e_{k l}\right)=\left\langle\left(e_{i j} \otimes e_{k l}\right) \xi, \xi\right\rangle=\frac{1}{n} \delta_{i k} \delta_{j l},
$$

that $\langle(\varphi(A) \otimes B) \xi, \xi\rangle=\operatorname{tr}\left(\varphi(A)^{t} B\right)$ for any $A, B \in M_{n}(\mathbb{C})$, where $\left\{e_{i j}\right\}$ is the set of matrix units of $M_{n}(\mathrm{C})$, tr is the normalized trace, and ${ }^{t} B$ is the transpose of $B$. Therefore, we have

$$
\begin{gathered}
\langle(\varphi(A) \otimes I) \xi, \xi\rangle=\operatorname{tr}\left(\varphi(A)^{t} I\right)=\operatorname{tr}(\varphi(A))=\operatorname{tr}(A), \\
\langle(\varphi(I) \otimes B) \xi, \xi\rangle=\operatorname{tr}\left(\varphi(I)^{t} B\right)=\operatorname{tr}\left({ }^{t} B\right)=\operatorname{tr}(B) .
\end{gathered}
$$

We define the map $\pi$ from $\operatorname{UCPT}(n)$ to $\Gamma(n)$ by

$$
\pi(\varphi)(A \otimes B)=\langle(\varphi(A) \otimes B) \xi, \xi\rangle .
$$

The map $\pi$ is known to be bijective (see Refs. 1, 7, and 8), but we give its proof for the completeness of the paper. In what follows, we identify a state $\rho$ with its density matrix $D_{\rho}$ and write $\operatorname{rank}(\rho):=\operatorname{rank}\left(D_{\rho}\right)$.

Theorem 2.2: The map $\pi$ from $\operatorname{UCPT}(n)$ to $\Gamma(n)$ defined above is bijective and preserves the convex structure. In particular, $\pi(\varphi)$ is an extremal point of $\Gamma(n)$ if and only if $\varphi$ is an extremal point of $U C P T(n)$. Moreover, $\operatorname{rank}(\pi(\varphi))$ is equal to $r(\varphi)$.

Proof: It is easy to see that $\pi$ is injective, and so we show that $\pi$ is surjective. Take a marginal tracial state $\rho$ and consider the decomposition of the corresponding density matrix $D_{\rho}$ into rank one positive operators which has the form $D_{\rho}=\sum_{i=1}^{k}\left|\zeta_{i}\right\rangle\left\langle\zeta_{i}\right|$ for some $\zeta_{i} \in \mathbb{C}^{n^{2}}=\mathbb{C}^{n} \otimes \mathbb{C}^{n}$. We write

$$
\zeta_{i}=\sum_{j=1}^{n} \zeta_{i j} \otimes e_{j} \in \mathbb{C}^{n} \otimes \mathbb{C}^{n}
$$

and define $v_{i} \in M_{n}(\mathbb{C})$ by $v_{i}\left(e_{j}\right)=\sqrt{n} \zeta_{i j}$ and put $\varphi=\sum_{i=1}^{k} v_{i}^{*} \cdot v_{i}$. Since $\varphi$ is completely positive and the domain of $\pi$ can be extended to the set of all completely positive maps, one can define a positive linear functional $\pi(\varphi)$ on $M_{n}(\mathrm{C}) \otimes M_{n}(\mathrm{C})$. Then we have 


$$
\begin{aligned}
D_{\pi(\varphi)} & =\sum_{i=1}^{k}\left|\left(v_{i} \otimes I\right) \xi\right\rangle\left\langle\left(v_{i} \otimes I\right) \xi\left|=\sum_{i=1}^{k}\right| \sum_{j=1}^{n}\left(\zeta_{i j} \otimes e_{j}\right)\right\rangle\left\langle\sum_{j=1}^{n}\left(\zeta_{i j} \otimes e_{j}\right)\right| \\
& =\sum_{i=1}^{k}\left|\zeta_{i}\right\rangle\left\langle\zeta_{i}\right|=D_{\rho}
\end{aligned}
$$

and hence $\pi(\varphi)=\rho$. Moreover, we have

$$
\begin{gathered}
\operatorname{tr}(\varphi(A))=\operatorname{tr}\left(\varphi(A)^{t} I\right)=\rho(A \otimes I)=\operatorname{tr}(A), \\
\operatorname{tr}\left(\varphi(I)^{t} B\right)=\rho(I \otimes B)=\operatorname{tr}(B)=\operatorname{tr}\left({ }^{t} B\right)
\end{gathered}
$$

for all $A, B \in M_{n}(\mathrm{C})$, which imply that $\varphi$ is a UCPT map, and hence $\pi$ is surjective.

It is obvious that $\pi$ preserves the convex structure.

Since $\left\{v_{i}\right\}$ is a linearly independent set, so is $\left\{\left(v_{i} \otimes I\right) \xi\right\}$. Thus, the density matrix,

$$
D_{\pi(\varphi)}=\sum_{i=1}^{k}\left|\left(v_{i} \otimes I\right) \xi\right\rangle\left\langle\left(v_{i} \otimes I\right) \xi\right|,
$$

has rank $k$, which implies $\operatorname{rank}(\pi(\varphi))=r(\varphi)$.

By Theorem 1 in Ref. 4, if $n \geq 3$, there exists an extremal point $\varphi$ of $\operatorname{UCPT}(n)$ with $r(\varphi)$ $\geq 2$ and hence $\varphi$ is a nonunitary map. Therefore, by Theorem 2.2 there exists an extremal marginal tracial state in $\Gamma(n)$ which is not pure.

Next we compute the maximal rank, denoted by $M R(n)$, of extremal marginal tracial states in $\Gamma(n)$. To this end, we collect some results on the extremal points of $\operatorname{UCPT}(n)$ and $\Gamma(n)$.

Definition 2.3: The pairs $\left(u_{1}, v_{1}\right), \ldots\left(u_{k}, v_{k}\right)$ of elements of a linear space are said to be bi-independent if

$$
\sum_{j=1}^{k} c_{j} u_{j}=0 \quad \text { and } \quad \sum_{j=1}^{k} c_{j} v_{j}=0
$$

imply $c_{j}=0$ for all $1 \leq j \leq k$. The $k$ pairs of elements are denoted by $u_{1}, \ldots, u_{k} ; v_{1}, \ldots, v_{k}$ (the relative order of the $u$ 's and $v$ 's being fixed).

Theorem 2.4: (Reference 4) Let $\varphi=\sum_{i=1}^{k} v_{i}^{*} \cdot v_{i}$, where $\left\{v_{i}\right\}$ is a linearly independent set with $\sum_{i=1}^{k} v_{i}^{*} v_{i}=\sum_{i=1}^{k} v_{i} v_{i}^{*}=I$. Then $\varphi$ is an extremal point of $U C P T(n)$ if and only if $\left\{v_{i} v_{j}^{*}\right\}_{i, j=1}^{k} ;\left\{v_{j}^{*} v_{i}\right\}_{i, j=1}^{k}$ is a bi-independent set.

Theorem 2.5: (Reference 6) Let $\rho$ be a marginal tracial state on $M_{n}(\mathrm{C}) \otimes M_{n}(\mathrm{C})$ and let $P_{\rho}$ be the support projection of $\rho$. Then $\rho$ is an extremal point of $\Gamma(n)$ if and only if $\left(P_{\rho}\left(M_{n}(\mathrm{C})\right.\right.$ $\left.\left.\otimes M_{n}(\mathrm{C})\right) P_{\rho}\right) \cap\left(\left(M_{n}(\mathrm{C}) \ominus \mathrm{CI}\right) \otimes\left(M_{n}(\mathrm{C}) \ominus \mathrm{CI}\right)\right)=\{0\}$.

Since the dimensions of $P_{\rho}\left(M_{n}(\mathrm{C}) \otimes M_{n}(\mathrm{C})\right) P_{\rho}, \quad\left(M_{n}(\mathrm{C}) \ominus \mathrm{CI}\right) \otimes\left(M_{n}(\mathrm{C}) \ominus \mathrm{CI}\right)$ and $M_{n}(\mathrm{C})$ $\otimes M_{n}(\mathrm{C})$ are $\operatorname{rank}(\rho)^{2},\left(n^{2}-1\right)^{2}$ and $n^{4}$, respectively, it follows from Theorem 2.5 that an upper bound on $M R(n)$ is given by

$$
M R(n) \leq \sqrt{2 n^{2}-1} .
$$

Every extremal point of $\Gamma(2)$ is pure, so that $M R(2)=1$. In the following, we compute $M R(3)$ and $M R(4)$.

Theorem 2.6: The maximal rank of extremal marginal tracial states in $\Gamma(3)$ is 4 .

Proof: From (1), we have $M R(3) \leq 4$. Hence all we need is to construct a map $\varphi$ which is extremal in $\operatorname{UCPT}(3)$ and has $r(\varphi)=4$. Let

$$
w_{1}=e_{11}, \quad w_{2}=e_{12}+\sqrt{2} e_{23}, \quad w_{3}=\sqrt{2} e_{21}+\sqrt{3} e_{32}, \quad w_{4}=e_{31}+\sqrt{2} e_{13} .
$$

Then we have 


$$
\sum_{i=1}^{4} w_{i} w_{i}^{*}=\sum_{i=1}^{4} w_{i}^{*} w_{i}=4 I,
$$

and hence $v_{i}=\frac{1}{2} w_{i}$ satisfies $\sum_{i=1}^{4} v_{i} v_{i}^{*}=\sum_{i=1}^{4} v_{i}^{*} v_{i}=I$. By using the equations

$$
\begin{array}{llll}
w_{1} w_{1}^{*}=e_{11} & w_{2} w_{1}^{*}=0 & w_{3} w_{1}^{*}=\sqrt{2} e_{21} & w_{4} w_{1}^{*}=e_{31} \\
w_{1} w_{2}^{*}=0 & w_{2} w_{2}^{*}=e_{11}+2 e_{22} & w_{3} w_{2}^{*}=\sqrt{3} e_{31} & w_{4} w_{2}^{*}=2 e_{12} \\
w_{1} w_{3}^{*}=\sqrt{2} e_{12} & w_{2} w_{3}^{*}=\sqrt{3} e_{13} & w_{3} w_{3}^{*}=2 e_{22}+3 e_{33} & w_{4} w_{3}^{*}=\sqrt{2} e_{32} \\
w_{1} w_{4}^{*}=e_{13} & w_{2} w_{4}^{*}=2 e_{21} & w_{3} w_{4}^{*}=\sqrt{2} e_{23} & w_{4} w_{4}^{*}=e_{33}+2 e_{11}
\end{array}
$$

and

$$
\begin{array}{llll}
w_{1}^{*} w_{1}=e_{11} & w_{2}^{*} w_{1}=e_{21} & w_{3}^{*} w_{1}=0 & w_{4}^{*} w_{1}=\sqrt{2} e_{31} \\
w_{1}^{*} w_{2}=e_{12} & w_{2}^{*} w_{2}=e_{22}+2 e_{33} & w_{3}^{*} w_{2}=2 e_{13} & w_{4}^{*} w_{2}=\sqrt{2} e_{32} \\
w_{1}^{*} w_{3}=0 & w_{2}^{*} w_{3}=2 e_{31} & w_{3}^{*} w_{3}=2 e_{11}+3 e_{22} & w_{4}^{*} w_{3}=\sqrt{3} e_{12} \\
w_{1}^{*} w_{4}=\sqrt{2} e_{13} & w_{2}^{*} w_{4}=\sqrt{2} e_{23} & w_{3}^{*} w_{4}=\sqrt{3} e_{21} & w_{4}^{*} w_{4}=e_{11}+2 e_{33},
\end{array}
$$

a simple calculation yields that $\Sigma_{i, j=1}^{4} a_{i j} w_{i} w_{j}^{*}=0$ and $\sum_{i, j=1}^{4} a_{i j} w_{j}^{*} w_{i}=0$ imply $a_{i j}=0$ for all $1 \leq i, j$ $\leq 4$. Therefore, $\left\{w_{i} w_{j}^{*}\right\} ;\left\{w_{j}^{*} w_{i}\right\}$ is a bi-independent set, and so is $\left\{v_{i} v_{j}^{*}\right\} ;\left\{v_{j}^{*} v_{i}\right\}$. Put $\varphi=\sum_{i=1}^{4} v_{i}^{*} \cdot v_{i}$. Then $\varphi$ is an extremal point of $\operatorname{UCPT}(3)$ with $r(\varphi)=4$ by Theorem 2.4, and $M R(3)=4$ follows

Theorem 2.7: The maximal rank of extremal marginal tracial states in $\Gamma(4)$ is 5 .

Proof: From (1), we have $M R(4) \leq 5$. Hence all we need is to construct a map $\varphi$ which is extremal in $U C P T(4)$ and has $r(\varphi)=5$. Let

$$
\begin{gathered}
w_{1}=e_{13}+e_{32}, \quad w_{2}=\sqrt{2} e_{24}+\sqrt{2} e_{43}, \quad w_{3}=\sqrt{2} e_{14}+\sqrt{3} e_{31}, \\
w_{4}=e_{21}+\sqrt{2} e_{42}, \quad w_{5}=e_{12}+e_{23} .
\end{gathered}
$$

Then it holds that

$$
\sum_{i=1}^{5} w_{i} w_{i}^{*}=\sum_{i=1}^{5} w_{i}^{*} w_{i}=4 I
$$

and hence $v_{i}=\frac{1}{2} w_{i}$ satisfy $\sum_{i=1}^{5} v_{i} v_{i}^{*}=\sum_{i=1}^{5} v_{i}^{*} v_{i}=I$. By the equations

$$
\begin{array}{lll}
w_{1} w_{1}^{*}=e_{11}+e_{33} & w_{2} w_{1}^{*}=\sqrt{2} e_{41} & w_{3} w_{1}^{*}=0 \\
w_{1} w_{2}^{*}=\sqrt{2} e_{14} & w_{2} w_{2}^{*}=2 e_{22}+2 e_{44} & w_{3} w_{2}^{*}=2 e_{12} \\
w_{1} w_{3}^{*}=0 & w_{2} w_{3}^{*}=2 e_{21} & w_{3} w_{3}^{*}=2 e_{11}+3 e_{33} \\
w_{1} w_{4}^{*}=\sqrt{2} e_{34} & w_{2} w_{4}^{*}=0 & w_{3} w_{4}^{*}=\sqrt{3} e_{32} \\
w_{1} w_{5}^{*}=e_{31}+e_{12} & w_{2} w_{5}^{*}=\sqrt{2} e_{42} & w_{3} w_{5}^{*}=0 \\
w_{4} w_{1}^{*}=\sqrt{2} e_{43} & w_{5} w_{1}^{*}=e_{13}+e_{21} & \\
w_{4} w_{2}^{*}=0 & w_{5} w_{2}^{*}=\sqrt{2} e_{24} & \\
w_{4} w_{3}^{*}=\sqrt{3} e_{23} & w_{5} w_{3}^{*}=0 & \\
w_{4} w_{4}^{*}=e_{22}+2 e_{44} & w_{5} w_{4}^{*}=\sqrt{2} e_{14} & \\
w_{4} w_{5}^{*}=\sqrt{2} e_{41} & w_{5} w_{5}^{*}=e_{11}+e_{22} &
\end{array}
$$

and 


$$
\begin{array}{lll}
w_{1}^{*} w_{1}=e_{33}+e_{22} & w_{2}^{*} w_{1}=0 & w_{3}^{*} w_{1}=\sqrt{2} e_{43}+\sqrt{3} e_{12} \\
w_{1}^{*} w_{2}=0 & w_{2}^{*} w_{2}=2 e_{44}+2 e_{33} & w_{3}^{*} w_{2}=0 \\
w_{1}^{*} w_{3}=\sqrt{2} e_{34}+\sqrt{3} e_{21} & w_{2}^{*} w_{3}=0 & w_{3}^{*} w_{3}=2 e_{44}+3 e_{11} \\
w_{1}^{*} w_{4}=0 & w_{2}^{*} w_{4}=\sqrt{2} e_{41}+2 e_{32} 2 & w_{3}^{*} w_{4}=0 \\
w_{1}^{*} w_{5}=e_{32} & w_{2}^{*} w_{5}=\sqrt{2} e_{43} & w_{3}^{*} w_{5}=\sqrt{2} e_{42} \\
w_{4}^{*} w_{1}=0 & w_{5}^{*} w_{1}=e_{23} & \\
w_{4}^{*} w_{2}=\sqrt{2} e_{14}+2 e_{23} & w_{5}^{*} w_{2}=\sqrt{2} e_{34} & \\
w_{4}^{*} w_{3}=0 & w_{5}^{*} w_{3}=\sqrt{2} e_{24} & \\
w_{4}^{*} w_{4}=e_{11}+2 e_{22} & w_{5}^{*} w_{4}=e_{31} & \\
w_{4}^{*} w_{5}=e_{13} & w_{5}^{*} w_{5}=e_{22}+e_{33}, &
\end{array}
$$

a simple calculation yields that $\sum_{i, j=1}^{5} a_{i j} w_{i} w_{j}^{*}=0$ and $\sum_{i, j=1}^{5} a_{i j} w_{j}^{*} w_{i}=0$ imply $a_{i j}=0$ for all $1 \leq i, j$ $\leq 5$. Therefore, $\left\{w_{i} w_{j}^{*}\right\} ;\left\{w_{j}^{*} w_{i}\right\}$ is a bi-independent set, and so is $\left\{v_{i} v_{j}^{*}\right\} ;\left\{v_{j}^{*} v_{i}\right\}$. Put $\varphi=\sum_{i=1}^{5} v_{i}^{*} \cdot v_{i}$. Then $\varphi$ is an extremal point of $\operatorname{UCPT}(4)$ with $r(\varphi)=5$ by Theorem 2.4 , and $M R(4)=5$ follows.

As to a lower bound on $M R(n)$ for $n>2$, we have the following estimate.

Theorem 2.8: The maximal rank of extremal marginal tracial states in $\Gamma(n)$ is at least $n$ for $n>2$.

Proof: Fix $n>2$ and we construct a map $\varphi$ which is extremal in $\operatorname{UCPT}(n)$ and has $r(\varphi)=n$. Let

$$
\begin{aligned}
& v_{1}=\sqrt{\frac{n-2}{n-1}} \sum_{j=2}^{n} e_{j j}, \\
& v_{i}=\frac{1}{\sqrt{n-1}}\left(e_{1 i}+e_{i 1}\right)
\end{aligned}
$$

for $2 \leq i \leq n$. Then we have

$$
\begin{gathered}
v_{1}^{*} v_{1}=\frac{n-2}{n-1} \sum_{j=2}^{n} e_{j j}, \\
v_{1}^{*} v_{j}=\frac{\sqrt{n-2}}{n-1} e_{j 1} \text { for } j \geq 2, \\
v_{j}^{*} v_{1}=\frac{\sqrt{n-2}}{n-1} e_{1 j} \text { for } j \geq 2, \\
v_{j}^{*} v_{j}=\frac{1}{n-1}\left(e_{11}+e_{j j}\right) \text { for } j \geq 2, \\
v_{j}^{*} v_{k}=\frac{1}{n-1} e_{j k} \text { for } j, k \geq 2 \text { and } j \neq k,
\end{gathered}
$$

and hence $\sum_{i=1}^{n} v_{i} v_{i}^{*}=\sum_{i=1}^{n} v_{i}^{*} v_{i}=I$. It follows from a simple calculation that $\left\{v_{j}^{*} v_{i}\right\}$ is a linearly independent set. Put $\varphi=\sum_{i=1}^{n} v_{i}^{*} \cdot v_{i}$. Then $\varphi$ is an extremal point of $\operatorname{UCPT}(n)$ with $r(\varphi)=n$ by Theorem 2.4, and $M R(n) \geq n$ follows. 


\section{DIAGONAL UCPT MAPS}

In this section, we give some properties of diagonal UCPT maps.

Definition 3.1: (Reference 4) A linear map $\varphi$ on $M_{n}(\mathrm{C})$ is diagonal if it has the form

$$
\varphi(A)=C \circ A
$$

for some $C \in M_{n}(\mathrm{C})$, where $C \circ A$ is the Schur product of $C$ and $A$.

Completely positive diagonal maps are characterized by the next proposition.

Proposition 3.2: (Reference 4) $\varphi$ is a completely positive diagonal map if and only if, in any representation,

$$
\varphi=\sum_{i=1}^{k} v_{i}^{*} \cdot v_{i}
$$

the matrices $v_{i}$ are diagonal.

In Ref. 4, it is shown that the maximal rank of extremal diagonal UCPT maps on $M_{n}(\mathrm{C})$ is at most $\sqrt{n}$. The next theorem shows that for any $a \in \mathbb{N}$ with $a^{2} \leq n$, we can construct an extremal diagonal UCPT map $\varphi$ with $r(\varphi)=a$. A marginal tracial state is called a diagonal marginal tracial state if the corresponding UCPT map is diagonal.

Theorem 3.3: Let $a \in \mathbb{N}$ be such that $a^{2} \leq n$. Then there are diagonal matrices $v_{1}, \ldots, v_{a}$ $\in M_{n}(\mathrm{C})$, such that the map

$$
\varphi(A)=\sum_{i=1}^{a} v_{i}^{*} A v_{i}, \quad A \in M_{n}(\mathbb{C})
$$

is an extremal UCPT map. Thus, $\varphi$ corresponds to an extremal diagonal marginal tracial state on $M_{n}(\mathrm{C}) \otimes M_{n}(\mathrm{C})$ of rank $a$.

Proof: If $a=1$, let $v_{1}$ be any diagonal unitary matrix in $M_{n}(\mathrm{C})$. So we suppose $a \geq 2$. Due to space limitation, in the following we write our diagonal matrices as row vectors with $n$ entries.

We first prove the theorem in the case that $a^{2}=n$. Choose $\theta_{1}, \ldots, \theta_{a} \in[0,2 \pi]$, such that the elements of the set $\left\{\theta_{i}-\theta_{j}: 1 \leq i \neq j \leq a\right\}$ are all distinct $\bmod 2 \pi$. Let

$$
\begin{aligned}
& b_{11}=\frac{1}{\sqrt{a}} e^{i \theta_{1}} \quad b_{12}=\frac{1}{\sqrt{a}} e^{2 i \theta_{1}} \quad \ldots \quad b_{1 l}=\frac{1}{\sqrt{a}} e^{l i \theta_{1}} \\
& b_{21}=\frac{1}{\sqrt{a}} e^{i \theta_{2}} \quad b_{22}=\frac{1}{\sqrt{a}} e^{2 i \theta_{2}} \quad \ldots \quad b_{2 l}=\frac{1}{\sqrt{a}} e^{l i \theta_{2}} \\
& \begin{array}{llll}
\vdots & \vdots & \vdots & \vdots
\end{array} \\
& b_{a 1}=\frac{1}{\sqrt{a}} e^{i \theta_{a}} \quad b_{a 2}=\frac{1}{\sqrt{a}} e^{2 i \theta_{a}} \quad \ldots \quad b_{a l}=\frac{1}{\sqrt{a}} e^{l i \theta_{a}},
\end{aligned}
$$

where $l=n-a=a^{2}-a$. Consider the following vectors of length $n=a^{2}$ :

$$
\begin{gathered}
v_{1}=(\overbrace{\left.1,0,0, \ldots, 0,0, b_{11}, b_{12}, \ldots b_{1 l}\right),}^{\text {length } a} \\
v_{2}=\left(0,1,0, \ldots, 0,0, b_{21}, b_{22}, \ldots b_{2 l}\right), \\
\vdots \\
v_{a}=\left(0,0,0, \ldots, 0,1, b_{a 1}, b_{a 2}, \ldots b_{a l}\right) .
\end{gathered}
$$

If we write the set $\left\{v_{i} v_{j}^{*}\right\}_{1 \leq i, j \leq a}$ as a list of $a^{2}$ row vectors, we have 


$$
\begin{gathered}
v_{1} v_{1}^{*}=(\overbrace{\left.1,0,0, \ldots, 0,0, \frac{1}{a}, \frac{1}{a}, \ldots, \frac{1}{a}\right)}^{\text {length } a} \overbrace{}^{\text {length } l}) \\
v_{1} v_{2}^{*}=\left(0,0,0, \ldots, 0,0, \frac{1}{a} e^{i\left(\theta_{1}-\theta_{2}\right)}, \frac{1}{a} e^{2 i\left(\theta_{1}-\theta_{2}\right)}, \ldots, \frac{1}{a} e^{l i\left(\theta_{1}-\theta_{2}\right)}\right), \\
v_{1} v_{3}^{*}=\left(0,0,0, \ldots, 0,0, \frac{1}{a} e^{i\left(\theta_{1}-\theta_{3}\right)}, \frac{1}{a} e^{2 i\left(\theta_{1}-\theta_{3}\right)}, \ldots, \frac{1}{a} e^{l i\left(\theta_{1}-\theta_{3}\right)}\right), \\
\vdots \\
v_{a} v_{a}^{*}=\left(0,0,0, \ldots, 0,1, \frac{1}{a}, \frac{1}{a}, \ldots, \frac{1}{a}\right) .
\end{gathered}
$$

Rearrange the rows in such a way that $v_{1} v_{1}^{*}, v_{2} v_{2}^{*}, \ldots, v_{a} v_{a}^{*}$ appear first, then we have the following $n \times n$ matrix:

$$
\left[\begin{array}{ccccccccccc}
1 & 0 & 0 & \cdots & 0 & 0 & \frac{1}{a} & \frac{1}{a} & \frac{1}{a} & \cdots & \frac{1}{a} \\
0 & 1 & 0 & \cdots & 0 & 0 & \frac{1}{a} & \frac{1}{a} & \frac{1}{a} & \cdots & \frac{1}{a} \\
\vdots & \vdots & \vdots & \vdots & \vdots & \vdots & \vdots & \vdots & \vdots & \vdots & \vdots \\
0 & 0 & 0 & \cdots & 0 & 1 & \frac{1}{a} & \frac{1}{a} & \frac{1}{a} & \cdots & \frac{1}{a} \\
0 & 0 & 0 & \cdots & 0 & 0 & \frac{1}{a} e^{i\left(\theta_{1}-\theta_{2}\right)} & \frac{1}{a} e^{2 i\left(\theta_{1}-\theta_{2}\right)} & \frac{1}{a} e^{3 i\left(\theta_{1}-\theta_{2}\right)} & \cdots & \frac{1}{a} e^{l i\left(\theta_{1}-\theta_{2}\right)} \\
\vdots & \vdots & \vdots & \vdots & \vdots & \vdots & \vdots & \vdots & \frac{1}{a} & \vdots & \vdots \\
0 & 0 & 0 & \cdots & 0 & 0 & \frac{1}{a} e^{i\left(\theta_{a}-\theta_{a-1}\right)} & \frac{1}{a} e^{2 i\left(\theta_{a}-\theta_{a-1}\right)} & \frac{1}{a} e^{3 i\left(\theta_{a}-\theta_{a-1}\right)} & \cdots & \frac{1}{a} e^{l i\left(\theta_{a}-\theta_{a-1}\right)}
\end{array}\right] .
$$

Note that this matrix has rank $n$ if and only if the lower corner $l \times l$ matrix has rank $l$. If one factors out $1 / a$ from each row of this $l \times l$ matrix, then the remaining matrix is

$$
\left[\begin{array}{cccc}
e^{i\left(\theta_{1}-\theta_{2}\right)} & e^{2 i\left(\theta_{1}-\theta_{2}\right)} & \cdots & e^{l i\left(\theta_{1}-\theta_{2}\right)} \\
e^{i\left(\theta_{1}-\theta_{3}\right)} & e^{2 i\left(\theta_{1}-\theta_{3}\right)} & \cdots & e^{l i\left(\theta_{1}-\theta_{3}\right)} \\
\vdots & \vdots & \vdots & \vdots \\
e^{i\left(\theta_{a}-\theta_{a-1}\right)} & e^{2 i\left(\theta_{a}-\theta_{a-1}\right)} & \cdots & e^{l i\left(\theta_{a}-\theta_{a-1}\right)}
\end{array}\right],
$$

which is a Vandermonde matrix with nonzero determinant because of the choice of $\theta_{1}, \ldots, \theta_{a}$. Consequently, $\left\{v_{i} v_{j}^{*}\right\}_{1 \leq i, j \leq a}$ is a linearly independent set and $\sum_{i=1}^{a} v_{i}^{*} v_{i}=\sum_{i=1}^{a} v_{i} v_{i}^{*}=I$. Thus, the diagonal map $\varphi=\sum_{i=1}^{a} v_{i}^{*} \cdot v_{i}$ is extremal in $U C P T(n)$.

If $a^{2}<n$, then add $n-a^{2}$ entries to the end of each of the vectors $v_{1}, \ldots, v_{a}$ to form 


$$
\begin{gathered}
v_{1}^{\prime}=v_{1} \oplus\left(c_{11}, c_{12}, \ldots, c_{1, n-a^{2}}\right), \\
v_{2}^{\prime}=v_{2} \oplus\left(c_{21}, c_{22}, \ldots, c_{2, n-a^{2}}\right), \\
\vdots \\
v_{a}^{\prime}=v_{a} \oplus\left(c_{a 1}, c_{a 2}, \ldots, c_{a, n-a^{2}}\right),
\end{gathered}
$$

where $\sum_{i=1}^{a}\left|c_{i j}\right|^{2}=1$ for all $1 \leq j \leq n-a^{2}$. Then we have

$$
\sum_{i=1}^{a} v_{i}^{\prime *} v_{i}^{\prime}=\sum_{i=1}^{a} v_{i}^{\prime} v_{i}^{\prime *}=I
$$

Furthermore, since $\left\{v_{i} v_{j}^{*}\right\}_{1 \leq i, j \leq a}$ is a linearly independent set, so is $\left\{v_{i}^{\prime} v_{j}^{\prime *}\right\}_{1 \leq i, j \leq a}$. Consequently, the mapping

$$
\varphi^{\prime}(A)=\sum_{i=1}^{a} v_{i}^{\prime *} A v_{i}^{\prime}, \quad A \in M_{n}(\mathrm{C})
$$

is an extremal UCPT map, and it corresponds to an extremal diagonal marginal tracial state on $M_{n}(\mathrm{C})$ of rank $a$.

Theorem 3.4: Extremal diagonal marginal tracial states of rank $a$ with $a^{2} \leq n$ are dense in the set of all diagonal marginal tracial states of rank a or less.

Proof: Let $\varphi$ be a diagonal UCPT map on $M_{n}(\mathrm{C})$ with

$$
\varphi(A)=\sum_{i=1}^{a} u_{i}^{*} A u_{i}
$$

for any $A \in M_{n}(\mathrm{C})$, where $u_{i}$ is possibly zero. Let $\psi$ be a diagonal UCPT map on $M_{n}(\mathrm{C})$ with

$$
\psi(A)=\sum_{i=1}^{a} v_{i}^{*} A v_{i}
$$

for any $A \in M_{n}(\mathrm{C})$. By Theorem 3.3, we may assume that $\left\{v_{i} v_{j}^{*}\right\}$ is a linearly independent set, so that $\psi$ is an extremal point of the set of all UCPT maps.

Fix $\varepsilon>0$ and put $w_{i}=u_{i}+\varepsilon v_{i}$. Since $\sum_{i=1}^{a} u_{i}^{*} u_{i}=I, \sum_{i=1}^{a} w_{i}^{*} w_{i}$ is invertible for sufficiently small $\varepsilon>0$, and hence one can define a diagonal UCPT map by

$$
\varphi_{\varepsilon}(A):=\left(\sum_{i=1}^{a} w_{i}^{*} w_{i}\right)^{-1} \sum_{i=1}^{a} w_{i}^{*} A w_{i}
$$

for any $A \in M_{n}(\mathrm{C})$. Since $\varphi_{\varepsilon}$ goes to $\varphi$ as $\varepsilon \rightarrow 0$, all we need is to verify that $\varphi_{\varepsilon}$ is an extremal point of the set of all UCPT maps. To this end, it suffices to prove that $\left\{w_{i} w_{j}^{*}\right\}$ is a linearly independent set for sufficiently small $\varepsilon>0$.

Since $w_{i} w_{j}^{*}$ is a diagonal matrix in $M_{n}(\mathrm{C})$, it can be considered a column vector in $\mathbb{C}^{n}$ and then $W=\left[w_{i} w_{j}^{*}\right]_{i, j=1}^{a}$ is an $n \times a^{2}$ matrix. Similarly, $U=\left[u_{i} u_{j}^{*}\right]_{i, j=1}^{a}$ and $V=\left[v_{i} v_{j}^{*}\right]_{i, j=1}^{a}$ can be considered $n \times a^{2}$ matrices.

Since $w_{i} w_{j}^{*}=\left(u_{i}+\varepsilon v_{i}\right)\left(u_{j}+\varepsilon v_{j}\right)^{*}=u_{i} u_{j}^{*}+\varepsilon\left(v_{i} u_{j}^{*}+u_{i} v_{j}^{*}\right)+\varepsilon^{2} v_{i} v_{j}^{*}$, if we put $X=\left[v_{i} u_{j}^{*}+u_{i} v_{j}^{*}\right]_{i, j=1}^{a}$, then we have

$$
W=U+\varepsilon X+\varepsilon^{2} V
$$

and hence 


$$
W^{*} W=U^{*} U+\varepsilon\left(U^{*} X+X^{*} U\right)+\varepsilon^{2}\left(U^{*} V+V^{*} U+X^{*} X\right)+\varepsilon^{3}\left(X^{*} V+V^{*} X\right)+\varepsilon^{4} V^{*} V
$$

The determinant of $W^{*} W$ is a polynomial of $\varepsilon$ of degree $4 a^{2}$ and the coefficient of $\varepsilon^{4 a^{2}}$ is the determinant of $V^{*} V$ which is not zero because $\left\{v_{i} v_{j}^{*}\right\}$ is a linearly independent set. Therefore, the equation $\left|W^{*} W\right|=0$ has at most $4 a^{2}$ solutions and $\left|W^{*} W\right|$ is not zero for sufficiently small $\varepsilon>0$. Since $\left\{w_{i} w_{j}^{*}\right\}$ is a linearly independent set if and only if $W^{*} W$ is invertible in $M_{a^{2}}(\mathrm{C})$, the proof is complete.

\section{ACKNOWLEDGMENTS}

The author is deeply grateful to Professor Geoffrey L. Price for helpful discussions. The proof of Theorem 3.3 is given by him. The author would like to express his gratitude to Professor Shôichirô Sakai and Professor Jun Kawabe for their useful advices. The author also appreciates a referee for valuable comments that helped to improve the final version of the paper.

${ }^{1}$ Arveson, W. B., "Subalgebras of $C^{*}$-algebras," Acta Math. 123, 141 (1969).

${ }^{2}$ Arveson, W. B., "The probability of entanglement," Commun. Math. Phys. 286, 283 (2009).

${ }^{3}$ Choi, M. D., "Completely positive linear maps on complex matrices," Linear Algebr. Appl. 10, 285 (1975).

${ }^{4}$ Landau, L. J. and Streater, R. F., "On Birkhoff's theorem for doubly stochastic completely positive maps on matrix algebras," Linear Algebr. Appl. 193, 107 (1993).

${ }^{5}$ Parthasarathy, K. R., "Extremal quantum states in coupled systems," Ann. Inst. Henri Poincare 41, 257 (2005).

${ }^{6}$ Price, G. L., and Sakai, S., "Extremal marginal tracial states in coupled systems," Operators and Matrices 1, 153 (2007).

${ }^{7}$ Rudolph, O., "On extremal quantum states of composite systems with fixed marginals," J. Math. Phys. 45, 4035 (2004).

${ }^{8}$ Størmer, E., "Extension of positive maps into $B(\mathcal{H})$," J. Funct. Anal. 66, 235 (1986). 